\title{
A robust adaptive beamforming method based on the matrix reconstruction against a large DOA mismatch
}

\author{
Julan Xie", Huiyong Li, Zishu He and Chaohai Li
}

\begin{abstract}
A novel adaptive beamforming algorithm against large direction-of-arrival (DOA) mismatch without using optimization toolboxes is proposed. In contrast to previous works, this new beamformer employs two reconstructed matrices, the interference-plus-noise covariance matrix and the desired signal-plus-noise covariance matrix, instead of their real sample covariance matrix, respectively. These reconstructed covariance matrices are used to obtain an orthogonal subspace, which is orthogonal to the interference subspace and contains the desired signal subspace. Without estimating the desired signal steering vector, an optimal weight can finally be solved by rotating this orthogonal subspace based on the output power of the desired signal maximization. This novel beamformer is able to keep a steady and outstanding performance when DOA mismatch has a large uncertainty level. Moreover, this algorithm overcomes the problem of the desired signal self-cancelation at high signal-to-noise ratio (SNR) while maintaining the good performance at low SNR.
\end{abstract}

Keywords: Robust adaptive beamformer; DOA mismatch; Covariance matrix reconstruction

\section{Introduction}

Adaptive beamforming is a classic problem in array signal processing and has broad application prospects in military and civilian applications. The conventional adaptive beamformers suppress the interference based on the exact knowledge of the desired signal steering vector. However, the presence of the desired signal component in the training data makes their performance very sensitive to the model mismatch [1-3], which arises due to imprecisely known wavefield propagation conditions, array perturbations, imperfectly calibrated arrays and finite sample effect. Whenever a model mismatch exists, these beamformers will suffer severe performance degradation. Therefore, the robust adaptive beamformer (RAB) has attracted more attention recently. Various RABs have been developed $[4,5]$.

One popular RAB category, the eigenspace-based beamforming (ESB) techniques [6], is based on eigendecomposition and uses the signal subspace. It suffers a high probability of subspace swap at low signal-to-noise ratio (SNR). Another well-known RAB category is the one using

\footnotetext{
* Correspondence: julanxie@uestc.edu.cn

School of Electronic Engineering, University of Electronic Science and Technology of China, Chengdu, Sichuan 611731, China
}

the so-called diagonal loading technique $[7,8]$, where a scaled identity matrix is added to the sample covariance matrix. The main disadvantage of this RAB category is that, there is no reliable way to choose the optimal diagonal loading factor in different scenarios. The third RAB category is based on the principle of the worst-case performance optimization $[9,10]$ and makes explicit use of an uncertainty set of the desired signal steering vector. However, it has been proved that this RAB category is equivalent to the second one [8]. Moreover, most beamformers of this $\mathrm{RAB}$ category are based on the second-order cone programming (SOCP) problem and needs to use some specific optimization toolboxes [11] to obtain the solution. Thus, their computation cost is high. This limits their practical implementation. Recently, an approach, where the key is estimating the real desired signal steering vector by using the region of the angular location of the desired signal steering vector, has been an intensive research topic [12-18]. For this $\mathrm{RAB}$ category technique, it chooses the weight vector by maximizing the output power under some restrictions without considering the worst-case performance optimization rule. However, most beamformers of this $\mathrm{RAB}$ category are based on a quadratically constrained 
quadratic programming (QCQP) problem, whose solution is obtained by using the convex optimization toolboxes such as CVX [19]. This also hits the wall of the computation complexity. In [17], Wei Zhang propose a novel method where the problem of finding the desired steering vector is an eigendecomposition problem that can be easily solved without any specific optimization software. However, they ignore the requirement that the estimate does not converge to any of the interference steering vectors and their linear combinations. This results in severe performance degradation when the SNR of desired signal is very small.

Most of the above-mentioned RABs suffer severe performance degradation when the desired signal has high SNR. Even the first RAB category also would fail to provide complete suppression of unwanted interferences when the power of desired signal is high. In [16], authors have proposed a robust beamformer based on the interference-plus-noise covariance matrix reconstruction and steering vector estimation. This beamformer performs well both at low and high SNRs. However, this beamformer estimates the steering vector by using the convex optimization software, which has a high computational cost. Furthermore, the inaccurate estimation leads to the output SNR loss, especially for a large direction-of-arrival (DOA) mismatch.

In this paper, we present a robust beamformer based on the matrix reconstruction for a large DOA mismatch. We reconstruct the interference-plus-noise covariance matrix and the desired signal-plus-noise covariance matrix, respectively, by using the Capon spectral estimator integrated over regions where the interference and desired signals are located, respectively. Based on these two reconstructed matrices, we can get an orthogonal subspace, which is orthogonal to the interference subspace and contains the desired signal subspace. We rotate this orthogonal subspace to obtain the optimal weight by maximizing the output power of desired signal. Numerical examples demonstrate that our beamformer has almost always equal value to the optimal value when DOA mismatch has a large uncertainty level and whenever the SNR level of the desired signal is low or high.

\section{The signal model}

Assume that an array of $M$ omni-directional antenna elements receives signals from multiple narrowband sources. The array observation $\boldsymbol{x}(k)$ at the time instant $k$ can be given by

$$
\boldsymbol{x}(k)=\boldsymbol{x}_{s}(k)+\boldsymbol{x}_{i}(k)+\boldsymbol{n}(k)
$$

where $\boldsymbol{x}_{s}(k), \boldsymbol{x}_{i}(k)$, and $\boldsymbol{n}(k)$ are the vectors of the desired signal, the interference, and the noise, respectively. The desired signal, the interference, and the noise components of the array observation $\boldsymbol{x}(k)$ are assumed to be statistically independent of each other. The desired signal can be modeled as $\boldsymbol{x}_{s}(k)=\boldsymbol{a}_{0} s(k)$, where $s(k)$ is the desired signal waveform and $\boldsymbol{a}_{0}$ is the associated steering vector.

The beamformer output can be written as

$$
y(k)=\boldsymbol{w}^{H} \boldsymbol{x}(k)
$$

where $\boldsymbol{w}$ is the complex weight vector for beamforming and $(\bullet)^{H}$ stands for the Hermitian transpose. If the steering vector $\boldsymbol{a}_{0}$ is known exactly, the optimal weight vector $\boldsymbol{w}$ can be achieved via maximizing the beamformer output signal-to-interference-plus-noise ratio (SINR)

$$
\operatorname{SINR}=\frac{\boldsymbol{w}^{H} \boldsymbol{R}_{s} \boldsymbol{w}}{\boldsymbol{w}^{H} \boldsymbol{R}_{i+n} \boldsymbol{w}}=\frac{\sigma_{s}^{2}\left|\boldsymbol{w}^{H} \boldsymbol{a}_{0}\right|}{\boldsymbol{w}^{H} \boldsymbol{R}_{i+n} \boldsymbol{w}}
$$

where $\boldsymbol{R}_{s}=E\left\{\boldsymbol{x}_{s}(k) \boldsymbol{x}_{s}^{H}(k)\right\}$ and $\boldsymbol{R}_{i+n}=E\left\{\left(\boldsymbol{x}_{i}(k)+\boldsymbol{n}(k)\right)\right.$ $\left.\left(\boldsymbol{x}_{\mathrm{i}}(k)+\boldsymbol{n}(k)\right)^{H}\right\}$ are the desired signal and the interferenceplus-noise covariance matrix, respectively. $E\{\bullet\}$ denotes the statistical expectation and $\sigma_{s}^{2}$ stands for the desired signal power. Since the exact interference-plus-noise covariance matrix $\boldsymbol{R}_{i+n}$ is hard to be separated from the covariance matrix $\boldsymbol{R}=\mathrm{E}\left\{\boldsymbol{x}(n) \boldsymbol{x}^{H}(n)\right\}=\boldsymbol{R}_{s}+\boldsymbol{R}_{i+n}$ in practice, it is replaced in (3) by the data sample covariance matrix

$$
\hat{\boldsymbol{R}}=\frac{1}{K} \sum_{k=1}^{k} \boldsymbol{x}(k) \boldsymbol{x}^{H}(k)
$$

where $K$ is the number of snapshots. Note that the sample covariance matrix contains the desired signal component. Hence, the estimate result, obtained by using $\hat{\boldsymbol{R}}$ is worse than the one using the interference-plus-noise covariance matrix $\boldsymbol{R}_{i+n}$.

The maximization problem (3), where the sample estimate $\hat{\boldsymbol{R}}$ is applied instead of $\boldsymbol{R}_{i+n}$, is mathematically equivalent to the MVDR sample matrix inversion (SMI) beamforming [20], which can be expressed as the following convex optimization problem:

$$
\min _{w} \boldsymbol{w}^{H} \hat{\boldsymbol{R}} \boldsymbol{w} \text { subject to } \boldsymbol{w}^{H} \boldsymbol{a}_{0}=1
$$

The solution of (5) is

$$
\boldsymbol{w}=\frac{\hat{\boldsymbol{R}}^{-1} \boldsymbol{a}_{0}}{\boldsymbol{a}_{0}^{H} \hat{\boldsymbol{R}}-1} \boldsymbol{a}_{0}
$$

The MVDR-SMI beamformer is known to be sensitive to any model mismatch of the desired signal steering vector. Mati Wax's researches [1,2] also have told us that both of the snapshots $K$ and the desired signal power affects the performance of the MVDR-SMI beamformer. Small sample size $K$ and high desired signal power would cause the degradation of the performance when there is DOA mismatch of the desired signal. It is easy to find that small sample size 
$K$ arouses a large gap between $\hat{\boldsymbol{R}}$ and $\boldsymbol{R}$. The high desired signal power leads to big difference between $\hat{\boldsymbol{R}}$ and $\boldsymbol{R}_{i+n}$.

Yujie $\mathrm{Gu}$ and Leshem have improved the MVDR-SMI beamformer by using a reconstructed matrix $\hat{\boldsymbol{R}}_{i+n}$ and an estimate desired signal steering vector instead of the sample estimate $\hat{\boldsymbol{R}}$ and the inexact desired signal steering vector, respectively. This new beamformer can acquire a good performance both at low and high SNRs. The interferenceplus-noise covariance matrix $\tilde{\boldsymbol{R}}_{i+n}$ was reconstructed as

$$
\tilde{\boldsymbol{R}}_{i+n}=\int_{\bar{\Theta}}\left(\frac{1}{\boldsymbol{a}^{H}(\theta) \hat{\boldsymbol{R}}^{-1} \boldsymbol{a}(\theta)}\right) \boldsymbol{a}(\theta) \boldsymbol{a}^{H}(\theta) d \theta
$$

where $\boldsymbol{a}(\theta)$ is the steering vector associated with a hypothetical direction $\theta$ based on the known array structure. $\Theta$ is an angular sector in which the desired signal is located and $\bar{\Theta}$ is the complement sector of $\Theta$. The estimate desired signal steering vector $\hat{\boldsymbol{a}}$ is obtained by solving the following problem

$$
\begin{aligned}
& \min _{\boldsymbol{e} \perp}\left(\overline{\boldsymbol{a}}+\boldsymbol{e}_{\perp}\right)^{H} \tilde{\boldsymbol{R}}_{i+n}^{-1}\left(\overline{\boldsymbol{a}}+\boldsymbol{e}_{\perp}\right) \\
& \text { subject to } \overline{\boldsymbol{a}}^{H} \boldsymbol{e}_{\perp}=0 \\
& \left(\overline{\boldsymbol{a}}+\boldsymbol{e}_{\perp}\right)^{H} \tilde{\boldsymbol{R}}_{i+n}^{-1}\left(\overline{\boldsymbol{a}}+\boldsymbol{e}_{\perp}\right) \leq \overline{\boldsymbol{a}}^{H} \tilde{\boldsymbol{R}}_{i+n}^{-1} \overline{\boldsymbol{a}}
\end{aligned}
$$

where the presumed steering vector $\overline{\boldsymbol{a}}$ is the inexact one and the estimate steering vector $\hat{\boldsymbol{a}}=\overline{\boldsymbol{a}}+\boldsymbol{e}_{\perp}$. However, the analysis in [15] has shown that $\overline{\boldsymbol{a}}^{H} \tilde{\boldsymbol{R}}_{i+n}^{-1} \overline{\boldsymbol{a}}$ may be the minimum. Thus, the constraint $\left(\overline{\boldsymbol{a}}+\boldsymbol{e}_{\perp}\right)^{H} \tilde{\boldsymbol{R}}_{i+n}^{-1}\left(\overline{\boldsymbol{a}}+\boldsymbol{e}_{\perp}\right) \leq \overline{\boldsymbol{a}}^{H}$ $\tilde{\boldsymbol{R}}_{i+n}^{-1} \overline{\boldsymbol{a}}$ would result to inaccurate estimation, which will result in the output SNR loss, especially for a large DOA mismatch.

\section{The proposed beamformer}

The proposed beamformer is based on the principle of maximizing output SINR. Recalling Equation (3), the following equation can be established

$$
\boldsymbol{R}_{s} \boldsymbol{w}_{\mathrm{SINR}_{\mathrm{opt}}}=\lambda \boldsymbol{R}_{i+n} \boldsymbol{w}_{\mathrm{SINR}_{\mathrm{opt}}}
$$

where $\boldsymbol{w}_{\text {SINR_opt }}$ denotes the optimal weight vector of the maximization problem (3) and $\lambda$ is a scale value equal to the maximum SINR. Owing to the existence of the noise, the interference-plus-noise covariance matrix $\boldsymbol{R}_{i+n}$ is always reversible. It is easy to be found that

$$
\boldsymbol{R}_{i+n}^{-1} \boldsymbol{R}_{s} \boldsymbol{w}_{\mathrm{SINR}_{\mathrm{opt}}}=\lambda \boldsymbol{w}_{\mathrm{SINR}_{\mathrm{opt}}}
$$

Apparently, the solution to the problem (10) is given by [3]

$$
\boldsymbol{w}_{\mathrm{SINR}_{\mathrm{opt}}}=\boldsymbol{v}\left\{\boldsymbol{R}_{i+n}^{-1} \boldsymbol{R}_{s}\right\}
$$

where $\boldsymbol{v}\{\bullet\}$ stands for the principal eigenvector of a matrix and $\lambda$ is the corresponding principal eigenvalue.
Since both the desired signal covariance matrices $\boldsymbol{R}_{s}$ and the interference-plus-noise covariance matrix $\boldsymbol{R}_{i+n}$ are unavailable even in signal-free applications, they can be replaced by two reconstructed matrices $\tilde{\boldsymbol{R}}_{s}$ and $\tilde{\boldsymbol{R}}_{i+n}$, respectively.

As assumed, $\bar{\Theta}$ is the complement sector of $\Theta$. It is clear that the DOAs of the interferences are located in the angular sector $\bar{\Theta}$. The reconstructed interference-plus-noise covariance matrix $\tilde{\boldsymbol{R}}_{i+n}$ can be obtained (see (7)) by using the Capon spatial spectrum. Similarly, the desired signalplus-noise covariance matrix $\tilde{\boldsymbol{R}}_{s}$ can be given by

$$
\tilde{\boldsymbol{R}}_{s}=\int_{\Theta}\left(\frac{1}{\boldsymbol{a}^{H}(\theta) \hat{\boldsymbol{R}}^{-1} \boldsymbol{a}(\theta)}\right) \boldsymbol{a}(\theta) \boldsymbol{a}^{H}(\theta) d \theta
$$

$\tilde{\boldsymbol{R}}_{i+n}$ collects all information on interference and noise in $\bar{\Theta}$. Hence, the effect of the desired signal is removed from the reconstructed covariance matrix $\tilde{\boldsymbol{R}}_{i+n} . \tilde{\boldsymbol{R}}_{s}$ gathers all information on desired signal and noise in $\Theta$. Consequently, the influence of the interferences is eliminated from the reconstructed covariance matrix $\tilde{\boldsymbol{R}}_{s}$. It is obvious that the steering vector of the desired signal and the interference signal lies in the subspace spanned by the columns of the principal eigenvectors of $\tilde{\boldsymbol{R}}_{s}$ and $\tilde{\boldsymbol{R}}_{i+n}$, respectively. Note that the Capon spatial spectrum peak is not a Dirac delta function. Therefore, unlike the rankone matrix $\boldsymbol{R}_{\mathrm{s}}$ in (3), $\tilde{\boldsymbol{R}}_{s}$ here is not rank-one matrix anymore.

An eigendecomposition of $\tilde{\boldsymbol{R}}_{i+n}$ results in a signal and noise subspace

$$
\tilde{\boldsymbol{R}}_{i+n}=\tilde{\boldsymbol{Q}}_{s} \tilde{\boldsymbol{\Xi}}_{s} \tilde{\boldsymbol{Q}}_{s}^{H}+\tilde{\boldsymbol{Q}}_{n} \tilde{\boldsymbol{\Xi}}_{n} \tilde{\boldsymbol{Q}}_{n}^{H}
$$

where $\tilde{\boldsymbol{Q}}_{s}$ and $\tilde{\boldsymbol{Q}}_{n}$ represent the signal and noise subspace eigenvectors and the diagonal matrices $\tilde{\boldsymbol{\Xi}}_{s}$ and $\tilde{\boldsymbol{\Xi}}_{n}$ include the signal subspace and noise subspace eigenvalues, respectively. Assume that the number of the interference signals is $L$ and $\boldsymbol{a}_{l}(l=1,2,3, \cdots, L)$ is the steering vector of the interference signal. It can be concluded that

$$
\begin{aligned}
\boldsymbol{a}_{l}^{H} \tilde{\boldsymbol{R}}_{i+n}^{-1} \tilde{\boldsymbol{R}}_{s} & =\boldsymbol{a}_{l}^{H}\left(\tilde{\boldsymbol{Q}}_{s} \tilde{\boldsymbol{\Xi}}_{s}^{-1} \tilde{\boldsymbol{Q}}_{s}^{H}+\tilde{\boldsymbol{Q}}_{n} \tilde{\boldsymbol{\Xi}}_{n}^{-1} \tilde{\boldsymbol{Q}}_{n}^{H}\right) \tilde{\boldsymbol{R}}_{s} \\
& =\boldsymbol{a}_{l}^{H} \tilde{\boldsymbol{Q}}_{s} \tilde{\boldsymbol{\Xi}}_{s}^{-1} \tilde{\boldsymbol{Q}}_{s}^{H} \tilde{\boldsymbol{R}}_{s}+\boldsymbol{a}_{l}^{H} \tilde{\boldsymbol{Q}}_{n} \tilde{\boldsymbol{\Xi}}_{n}^{-1} \tilde{\boldsymbol{Q}}_{n}^{H} \tilde{\boldsymbol{R}}_{s}, l=1,2, \cdots, L
\end{aligned}
$$

As discussed, it is clear that

$$
\boldsymbol{a}_{l}^{H} \tilde{\boldsymbol{Q}}_{n}=0, l=1,2, \cdots, L
$$

Thus, the second term in (14) becomes $\boldsymbol{a}_{l}^{H} \tilde{\boldsymbol{Q}}_{n} \tilde{\boldsymbol{\Xi}}_{n}^{-1} \tilde{\boldsymbol{Q}}_{n}^{H}$ $\tilde{\boldsymbol{R}}_{s}=0$. When the power of the interference is strong, $\tilde{\boldsymbol{\Xi}}_{s}^{-1} \approx 0$ leads to 


$$
\boldsymbol{a}_{l}^{H} \tilde{\boldsymbol{Q}}_{s} \tilde{\boldsymbol{\Xi}}_{s}^{-1} \tilde{\boldsymbol{Q}}_{s}^{H} \tilde{\boldsymbol{R}}_{s} \approx 0, l=1,2, \cdots, L
$$

Combine Equations (14), (15) and (16), a final result is obtained as

$$
\boldsymbol{a}_{l}^{H} \tilde{\boldsymbol{R}}_{i+n}^{-1} \tilde{\boldsymbol{R}}_{s} \approx 0, l=1,2, \cdots, L
$$

Perform the eigenvalue decomposition on the matrix $\tilde{\boldsymbol{R}}_{f}=\tilde{\boldsymbol{R}}_{i+n}^{-1} \tilde{\boldsymbol{R}}_{s}$ as

$$
\begin{aligned}
\tilde{\boldsymbol{R}}_{f} & =\boldsymbol{U}_{s} \boldsymbol{\Lambda}_{s} \boldsymbol{U}_{s}^{H}+\boldsymbol{U}_{n} \boldsymbol{\Lambda}_{n} \boldsymbol{U}_{n}^{H} \\
& \approx \boldsymbol{U}_{s} \boldsymbol{\Lambda}_{s} \boldsymbol{U}_{s}^{H}
\end{aligned}
$$

where $\boldsymbol{U}_{s}$ and $\boldsymbol{U}_{n}$ denote the signal and noise subspace eigenvectors and the diagonal matrices $\Lambda_{s}$ and $\Lambda_{n}$ include the signal subspace and noise subspace eigenvalues, respectively. The finite sample snapshot number leads to $\Lambda_{n} \neq 0$ but $\Lambda_{n} \approx 0$. Therefore, $\tilde{\boldsymbol{R}}_{f}$ is approximately equal to $\boldsymbol{U}_{s} \boldsymbol{\Lambda}_{s} \boldsymbol{U}_{s}^{H}$. As explained above, thanks to the multiple-rank matrix $\tilde{\boldsymbol{R}}_{s}$, the subspace $\boldsymbol{U}_{s}$ is not rank-one matrix yet. Due to Equation (17), the subspace $\boldsymbol{U}_{s}$ satisfies the following equation

$$
\boldsymbol{a}_{l}^{H} \boldsymbol{U}_{s}=0, l=1,2, \cdots, L
$$

Moreover, the origin of $\boldsymbol{U}_{s}$ indicates that this subspace contains the desired signal subspace. The characters of $\boldsymbol{U}_{s}$ relating to the interference subspace and desired signal subspace allow the beamformer weight vector to be constructed as

$$
\boldsymbol{w}=\boldsymbol{U}_{s} \boldsymbol{r}
$$

where $r$ is the rotating vector. Then, it is easy to find that

$$
\boldsymbol{w}^{H} \boldsymbol{a}_{l}=\boldsymbol{r}^{H} \boldsymbol{U}_{s}^{H} \boldsymbol{a}_{l} \approx 0, l=1,2, \cdots, L
$$

Rewriting Equation (3) by using $\tilde{\boldsymbol{R}}_{s}$ and $\tilde{\boldsymbol{R}}_{i+n}$ instead of $\boldsymbol{R}_{s}$ and $\boldsymbol{R}_{i+n}$, respectively, another expression of SINR can be written as

$$
\operatorname{SINR}_{\mathrm{Rec}}=\frac{\boldsymbol{w}^{H} \tilde{\boldsymbol{R}}_{s} \boldsymbol{w}}{\boldsymbol{w}^{H} \tilde{\boldsymbol{R}}_{i+n} \boldsymbol{w}}
$$

Let us observe the denominator of Equation (22) first. Recalling Equations (13) and (20), it can be concluded

$$
\begin{aligned}
& \boldsymbol{w}^{H} \tilde{\boldsymbol{R}}_{i+n} \boldsymbol{w}=\boldsymbol{r}^{H} \boldsymbol{U}_{s}^{H}\left(\tilde{\boldsymbol{Q}}_{s} \tilde{\boldsymbol{\Xi}}_{s} \tilde{\boldsymbol{Q}}_{s}^{H}+\tilde{\boldsymbol{Q}}_{n} \tilde{\boldsymbol{\Xi}}_{n} \tilde{\boldsymbol{Q}}_{n}^{H}\right) \boldsymbol{U}_{s} \boldsymbol{r} \\
& \quad=\boldsymbol{r}^{H} \boldsymbol{U}_{s}^{H} \tilde{\boldsymbol{Q}}_{s} \tilde{\boldsymbol{\Xi}}_{s} \tilde{\boldsymbol{Q}}_{s}^{H} \boldsymbol{U}_{s} \boldsymbol{r}+\boldsymbol{r}^{H} \boldsymbol{U}_{s}^{H} \tilde{\boldsymbol{Q}}_{n} \tilde{\boldsymbol{\Xi}}_{n} \tilde{\boldsymbol{Q}}_{n}^{H} \boldsymbol{U}_{s} \boldsymbol{r}
\end{aligned}
$$

It is known that

$$
\boldsymbol{a}_{l}=\tilde{\boldsymbol{Q}}_{s} \boldsymbol{e}_{l}, l=1,2, \cdots, L
$$

where $\boldsymbol{e}_{l}$ is the rotating vector. According to (19), the first term of (23) becomes $\boldsymbol{r}^{H} \boldsymbol{U}_{s}^{H} \tilde{\boldsymbol{Q}}_{s} \tilde{\boldsymbol{\Xi}}_{s} \tilde{\boldsymbol{Q}}_{s}^{H} \boldsymbol{U}_{s} \boldsymbol{r} \approx 0$. The second term of (23) can be expressed as

$$
\begin{aligned}
\boldsymbol{r}^{H} \boldsymbol{U}_{s}^{H} \tilde{\boldsymbol{Q}}_{n} \tilde{\boldsymbol{\Xi}}_{n} \tilde{\boldsymbol{Q}}_{n}^{H} \boldsymbol{U}_{s} \boldsymbol{r} & =\boldsymbol{r}^{H} \boldsymbol{B}^{H} \tilde{\boldsymbol{\Xi}}_{n} \boldsymbol{B} \boldsymbol{r} \\
& =\sum \gamma_{i} \boldsymbol{r}^{H} \boldsymbol{b}_{i}^{H} \boldsymbol{b}_{i} \boldsymbol{r} \\
& =\sum \gamma_{i} \alpha_{i}
\end{aligned}
$$

where $\boldsymbol{B}=\tilde{\boldsymbol{Q}}_{n}^{H} \boldsymbol{U}_{s}$ and $\boldsymbol{b}_{i}$ is the $i$ th column of the matrix $\boldsymbol{B}$. $\alpha_{i}=\boldsymbol{r}^{H} \boldsymbol{b}_{i}^{H} \boldsymbol{b}_{i} \boldsymbol{r} \geq 0$ and $\gamma_{i}$ is the $i$ th diagonal value of matrix $\tilde{\boldsymbol{\Xi}}_{n}$ and is very small. Thus, the second term of (23) can be ignored. The derivation shows that $\boldsymbol{w}^{H} \tilde{\boldsymbol{R}}_{i+n} \boldsymbol{w}$ can achieve a minimum value if we choose the beamformer vector in Equation (20). Then, the $\operatorname{SINR}_{\text {Rec }}$ maximization problem is transformed into the following optimization problem

$$
\max _{w} \boldsymbol{w}^{H} \tilde{\boldsymbol{R}}_{s} \boldsymbol{w}^{w=U r}=\max _{r} \boldsymbol{r}^{H} \boldsymbol{U}_{s}^{H} \tilde{\boldsymbol{R}}_{s} \boldsymbol{U}_{s} \boldsymbol{r}
$$

Obviously, the solution to the above problem is

$$
\boldsymbol{r}_{\text {Rec }}=M\left\{\boldsymbol{R}_{u}\right\}
$$

where $\boldsymbol{R}_{u}=\boldsymbol{U}_{s}^{H} \tilde{\boldsymbol{R}}_{s} \boldsymbol{U}_{s}$ and $M\{\bullet\}$ stands for the eigenvector of a matrix corresponding to the maximum eigenvalue. Substituting (27) into (20), the final optimal beamformer vector can be modelled as

$$
\boldsymbol{w}_{\text {Rec }}=\boldsymbol{U}_{s} \boldsymbol{r}_{\text {Rec }}
$$

The steps involved in the proposed beamformer can be summarized below:

(1)Compute the sample covariance matrix $\hat{\boldsymbol{R}}$ by using (4).

(2) Reconstruct the interference-plus-noise covariance matrix $\tilde{\boldsymbol{R}}_{i+n}$ and desired signal-plus-noise covariance matrix $\tilde{\boldsymbol{R}}_{s}$ according to Equations (7) and (12), respectively.

(3) Estimate the orthogonal subspace $\boldsymbol{U}_{s}$ via an eigenvalue decomposition of $\tilde{\boldsymbol{R}}_{f}=\tilde{\boldsymbol{R}}_{i+n}^{-1} \tilde{\boldsymbol{R}}_{s}(\operatorname{see}(18))$.

(4)Calculate the rotating vector $\boldsymbol{r}_{\text {Rec }}$ by using (27).

(5)Obtain the beamformer weight vector $\boldsymbol{w}_{\text {Rec }}$ with Equation (28).

The computation complexity of the reconstruction of the interference-plus-noise covariance matrix and the desired signal-plus-noise covariance matrix is $O\left(M^{2}\right)$ ), where $J$ is the number of sampled points in the DOA region. The computation complexity of the solution the final weight vector is dominated by the eigenvalue decomposition of $\tilde{\boldsymbol{R}}_{f}$, which is $O\left(M^{3}\right)$. Therefore, the total computation complexity of the proposed algorithm is $O\left(M^{2} \gamma\right)+O\left(M^{3}\right)$. The SOCP- or QCQP-based beamformers also make the twostep method equivalent to the proposed method. The matrix inversion operation of the simplest SMI beamformer has a complexity of $O\left(M^{3}\right)$. The solution of the SOCP or QCQP problem to obtain the final optimal weight vector 
has at least the complexity of $O\left(M^{3.5}\right)$. Hence, the total computation complexity of the SOCP/QCQP-based bemformers is not less than $O\left(M^{3}\right)+O\left(M^{3.5}\right)$. If the SOCP- or QCQP-based beamformers estimate the real desired signal steering vector by using the region of the angular location of the desired signal steering vector, their computation complexity is not less than $O\left(M^{2}\right)+O\left(M^{3.5}\right)$. This computation complexity is more than our proposed beamformer. Typically, $J>>$ M. There is $O\left(M^{2}\right)>O\left(M^{3}\right)$. However, if some priori information is used, the number of sampled points in the DOA region $J$ is able to be chosen to make $O$ $\left(M^{2}\right)<O\left(M^{3.5}\right)$. Overall, the proposed beamformer has a slight advantage to the SOCP- or QCQP-based beamformers in the view of the computation complexity. However, unlike the SOCP- or QCQP-based methods, the proposed method has an important advantage for being more easily implemented without any specific optimization software.

\section{Simulation results}

A uniform linear array of 10 sensors with half interelement spacing is employed. Additive noise in each antenna element is modeled as spatially and temporally independent complex Gaussian noise. Two interferences, which have the same interference-to-noise ratio (INR) of $30 \mathrm{~dB}$, are impinging on the array from directions $-30^{\circ}$ and $50^{\circ}$, respectively. The desired signal, assumed to be a plane wave from the presumed direction $\theta_{s}=5^{\circ}$, is always present in the training data. The possible angular sector of the desired signal is set to be $\Theta=\left[\theta_{s}-7^{\circ}, \theta_{s}+7^{\circ}\right]$. All results are averaged, based on 200 independent simulation runs.

The performance of the proposed algorithm is compared with the sample matrix inversion (SMI) beamformer, the eigenspace-based beamformer (ESB), the reconstruction-estimation (Rec-est.) beamformer [16], the Capon-estimation (Capon-est.) beamformer [17], and the Capon-estimation based on little information (Capon-est.-L) beamformer [15]. The dimension of the signal-plus-interference subspace is assumed to be always estimated correctly for the ESB. The CVX Matlab toolbox is used for solving the optimization problem in [15] and [16]. The number of the columns of the orthogonal subspace $\boldsymbol{U}_{s}$ for Capon-estimation (Capon-est.) beamformer in [17] is chosen as 4. Four principal eigenvectors of $\tilde{\boldsymbol{R}}_{f}$ corresponding to the four largest eigenvalues have been used in the proposed method.

Example 1: The beampattern of beamformers In this example, the resultant beampattern of the beamformers is considered. The snapshots number is 200 . A look direction mismatch of $-7^{\circ}$ is assumed. This means that the real DOA of the desired signal is $-2^{\circ}$. The SNR of the desired signal is $15 \mathrm{~dB}$. Array beampatterns of each beamformer are shown in Figure 1. It can be seen from Figure 1 that all these beamformers have deep nulls at DOAs of interferences. However, only the proposed beamformer and ESB form the main lobe in the correct look direction. For the SMI beamformer, the high desired signal SNR and large DOA mismatch together cause the appearance of the nulling in the real DOA of the desired signal. For the Rec-est. beamformer, the inaccurate estimation of the desired signal steering vector brings about an erroneous look direction. For the Capon-est. beamformer and the Capon-est.-L beamformer, the high desired signal SNR makes their main lobes point to the incorrect look directions.

Example 2: The output SINR versus the number of snapshots In the second example, the effect of the number of snapshots on the output SINR of beamformers is studied. The random DOA mismatch of the desired signal are uniformly distributed in $\left[-7^{\circ}, 7^{\circ}\right]$. That is to say, the DOA of the signal is uniformly distributed at $\left[-2^{\circ}, 12^{\circ}\right]$. The SNR of the desired signal is still $15 \mathrm{~dB}$. Here, the random DOA of the signal changes from run to run but remains fixed from snapshot to snapshot. The output SINR of the aforementioned methods versus the number of snapshots is compared in Figure 2. As shown, the proposed beamformer is always the closest one to the optimal SINR and enjoys much faster convergence rates rather than other beamformers except the Rec-est. beamformer. Although the Recest. beamformer has the same convergence rates with the proposed beamformer, its output SINR is always lower than the proposed one. The ESB, whose convergence rate is nearly same with the Capon-est. beamformer but lower than the SMI beamformer and the Capon-est-L. beamformer, always provides a higher output SINR than others except the proposed one and the Rec-est. beamformer.

Example 3: The output SINR versus the desired signal SNR Recalling Equation (3), the following equation can be established

$$
\mathrm{SINR}=\frac{\boldsymbol{w}^{H} \boldsymbol{R}_{s} \boldsymbol{w}}{\boldsymbol{w}^{H} \boldsymbol{R}_{i+n} \boldsymbol{w}}=\frac{\sigma_{s}^{2}\left|\boldsymbol{w}^{H} \boldsymbol{a}_{0}\right| / \sigma^{2}}{\boldsymbol{w}^{H} \boldsymbol{R}_{i+n} \boldsymbol{w} / \sigma^{2}}
$$

where $\sigma^{2}=E\left\{\boldsymbol{n}(\mathrm{k}) \boldsymbol{n}^{H}(k)\right\}$ is the power of the noise. Here, the output SNR and output interference-to-noise ratio (INR) are defined as

$$
\begin{gathered}
\mathrm{SNR}_{\text {output }}=\sigma_{s}^{2}\left|\boldsymbol{w}^{H} \boldsymbol{a}_{0}\right| / \sigma^{2}=\left|\boldsymbol{w}^{H} \boldsymbol{a}_{0}\right| \mathrm{SNR} \\
\mathrm{INR}_{\text {output }}=\boldsymbol{w}^{H} \boldsymbol{R}_{i+n} \boldsymbol{w} / \sigma^{2} \\
=\sum_{l=0}^{L}\left(P_{l}^{2} / \sigma^{2}\right) \boldsymbol{w}^{H} \boldsymbol{a}_{l} \boldsymbol{a}_{l}^{H} \boldsymbol{w}+\boldsymbol{w}^{H} \boldsymbol{w} \\
=\sum_{l=0}^{L} \mathrm{INR}_{l} \boldsymbol{w}^{H} \boldsymbol{a}_{l} \boldsymbol{a}_{l}^{H} \boldsymbol{w}+\boldsymbol{w}^{H} \boldsymbol{w}
\end{gathered}
$$

Apparently, $\operatorname{SINR}=\mathrm{SNR}_{\text {output }} / \mathrm{INR}_{\text {output }}$. In this example, the output SINR, the output SNR and the output 




Figure 1 The beampattern of beamformers.

INR versus different SNR of the desired signal are all given out. By observing these results, the process that the desired signal SNR how to affect the performance of each beamformer can be found out. Here, the look direction is still randomly and uniformly distributed at $\left[-2^{\circ}, 12^{\circ}\right]$. Hence, the random DOA mismatch of the desired signal is still uniformly distributed in $\left[-7^{\circ}, 7^{\circ}\right]$. The desired signal SNR varies from -20 to $50 \mathrm{~dB}$. The snapshot number is assumed to be 500 .

As deduced in the part 3, the proposed beamformer suppresses the interference due to the fact that the steering vector of the interference is approximately orthogonal to

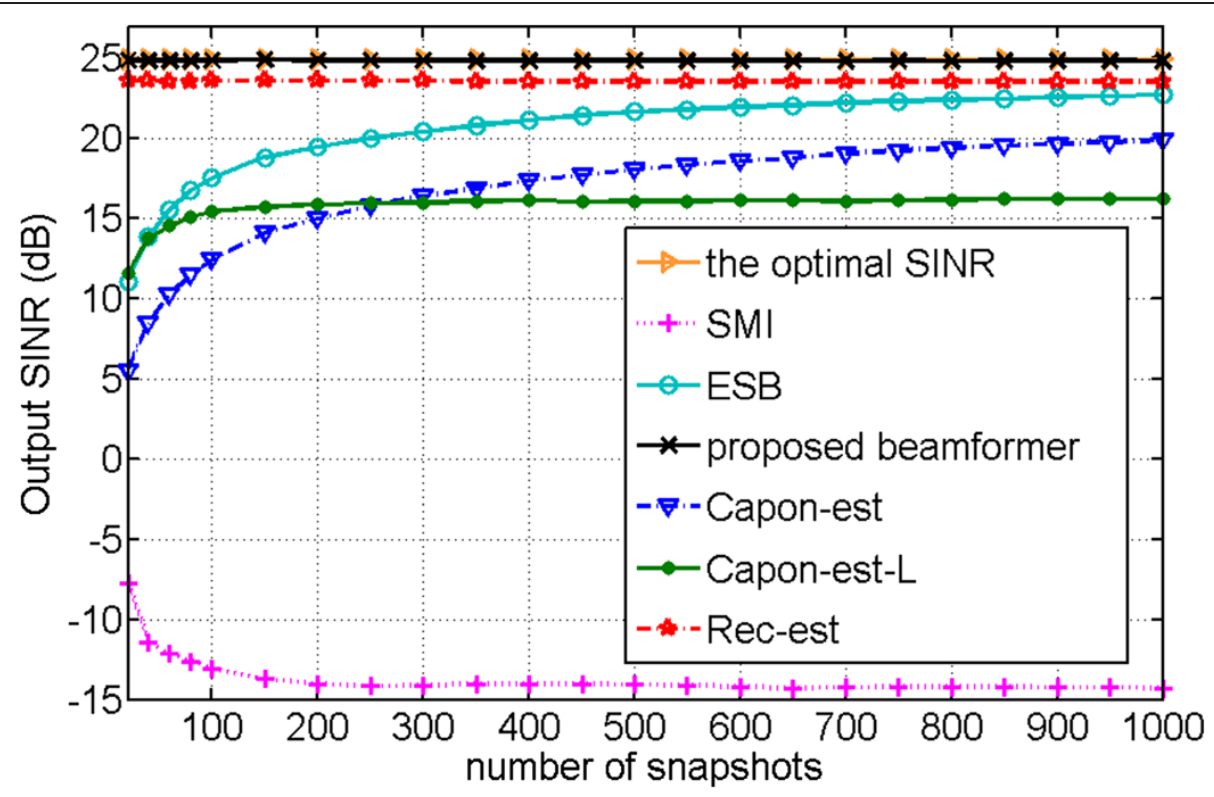

Figure 2 The output SINR versus the number of snapshots. 


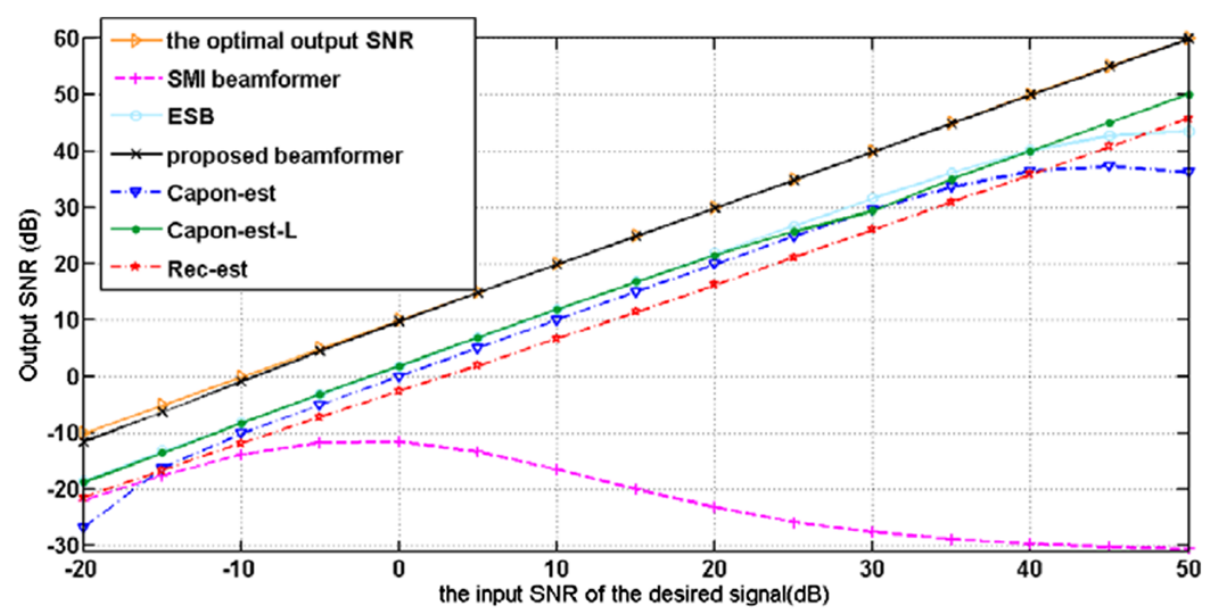

(a)



(b)

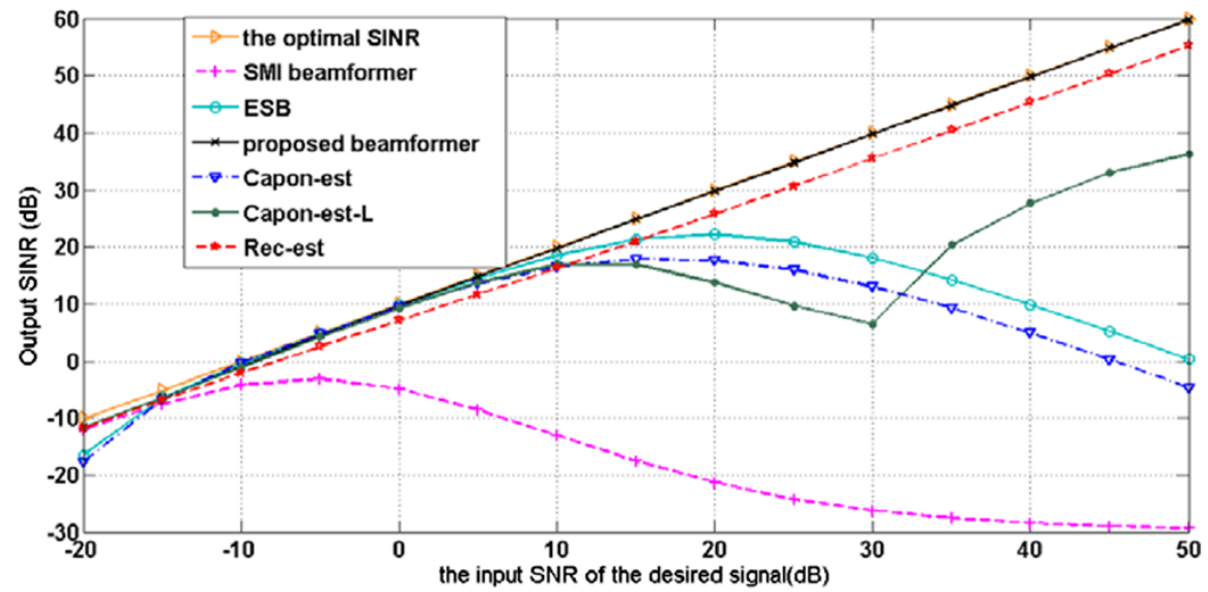

(c)

Figure 3 Output SNR (a), output INR (b), and output SINR (c) versus input SNR of desired signal. 




(a)

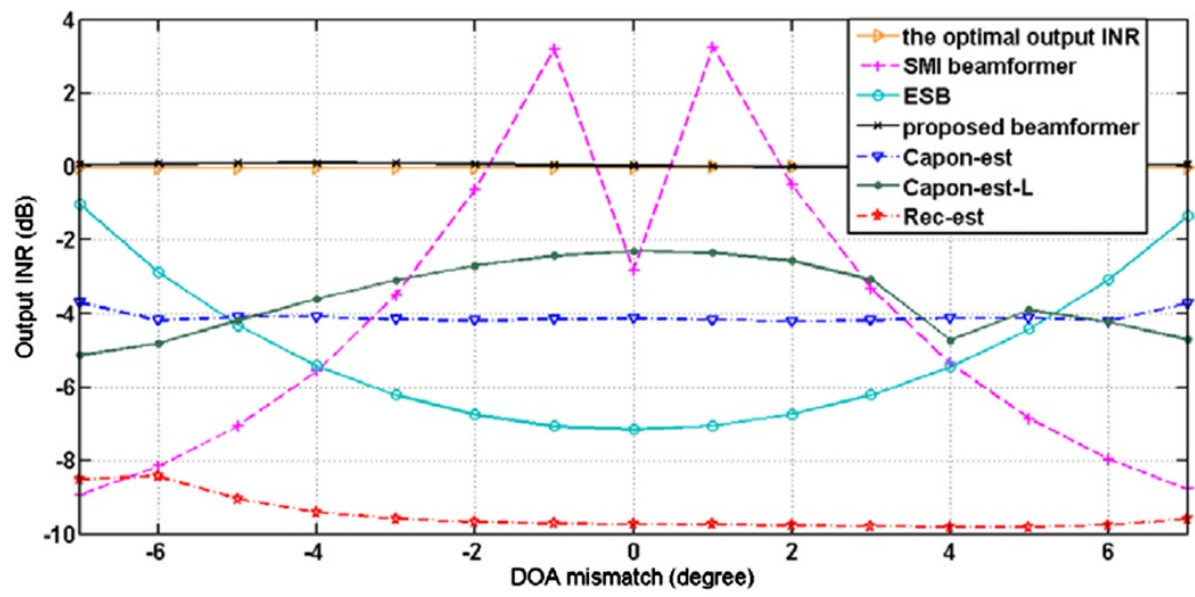

(b)

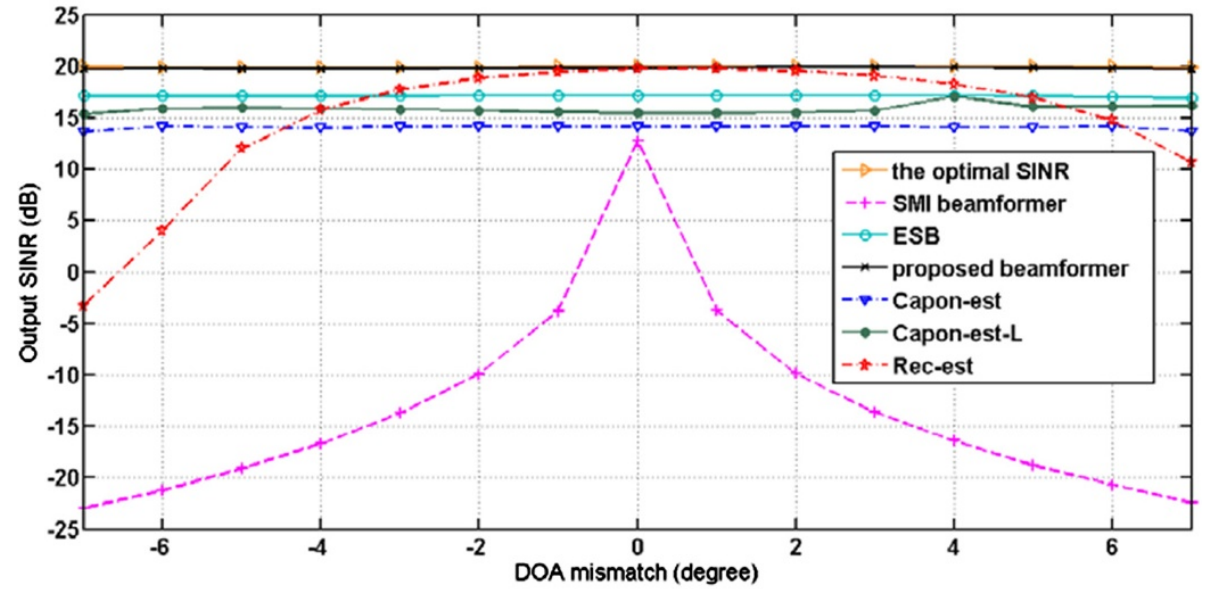

(c)

Figure 4 Output SNR (a), output INR (b), and output SINR (c) versus DOA mismatch of desired signal. 
the reconstruction matrix $\tilde{\boldsymbol{R}}_{f}=\tilde{\boldsymbol{R}}_{i+n}^{-1} \tilde{\boldsymbol{R}}_{s}$. For the optimal beamformer, the steering vector of the interference is approximately orthogonal to the matrix $\boldsymbol{R}_{f}=\boldsymbol{R}_{i+n}^{-1} \boldsymbol{R}_{s}$. It implies that the proposed beamformer and the optimal beamformer have nearly the same principle and ability of the interference suppression. The results in Figure $3 \mathrm{~b}$ investigate this saying. From Figure 3a, it can be found that the output SNR of the proposed one is quite close to the optimal beamformer in a large range from -20 to $50 \mathrm{~dB}$. The optimal weight maximizing the output SINR can be considered as a result of maximizing the output SNR under the premise of its approximately orthogonal to the steering vector of the interference signal. The proposed weight is obtained by using the same scheme. Therefore, the proposed beamformer produce an output SNR quite close to the optimal beamformer. As we know, the reconstructed matrix $\tilde{\boldsymbol{R}}_{s}$ is constructed by using Capon spectral estimator integrated over regions where desired signals are located. Hence, when the SNR is very small, the spectral peak, obtained by using reconstructed matrix $\tilde{\boldsymbol{R}}_{s}$, corresponding to the real DOA of the desired signal is quite flat. This causes an inaccurate estimate of the rotating vector $\boldsymbol{r}$, which leads to a slight worse result than the optimal beamformer. The joint action of the output SNR and the output INR results in that the proposed beamformer is always the closest one to the optimal SINR in a large range from -20 to $50 \mathrm{~dB}$ (see Figure 3c).

Because of the removing of the desired signal component from the covariance matrix, the output INR of the Rec-est. beamformer is not much sensitive to the desired signal SNR and can always follow the trend of the optimal beamformer. Moreover, the constraint of $\boldsymbol{w}^{H} \hat{\boldsymbol{a}}=1$ makes the value of $\boldsymbol{w}^{H} \boldsymbol{w}$ very small, which gives raise to that the output INR is smaller than the optimal beamformer and the proposed beamformer. However, due to the inaccurate estimation of the steering vector, the output SNR of the Recest. beamformer is inferior to the proposed one. Therefore, the final output SINR of the Rec-est. beamformer is smaller than the proposed one. The performance of the ESB, the Capon-est. beamformer and the Capon-est-L. beamformer can keep quite close to the optimal SINR in a range from -15 to $10 \mathrm{~dB}$ but degrade when SNR is higher than $20 \mathrm{~dB}$. This is because their interference suppression becomes worse versus the increase of the SNR. For the SMI beamformer, as shown in Figure 3, the output SNR decreases and the output INR increases when the high desired signal SNR and large DOA mismatch appear at the same time. Thus, the performance of the SMI beamformer would degrade versus the increase of the SNR.

Example 4: The output SINR versus DOA mismatch In the last example, the output SINR of beamformers versus different DOA mismatches is considered. Same with example 3, the output SNR results and output INR results are also presented. SNR is assumed to be $10 \mathrm{~dB}$ and the number of snapshots is chosen as 200. The DOA mismatch is uniformly distributed at $\left[-7^{\circ}, 7^{\circ}\right]$. Results are presented in Figure 4. As explained in example 3, the principle of obtaining the proposed beamformer imitates that of the optimal beamformer. When $\mathrm{SNR}=10 \mathrm{~dB}$, the proposed beamformer has nearly the same output SNR and INR. Hence, it is easy to find that the proposed algorithm always provides an output SINR almost equal to the optimal value when DOA mismatch has a large uncertainty level. The Rec-est. beamformer is strongly affected by the DOA mismatch level and so does the SMI beamformer. For the Rec-est. beamformer, the imprecise estimation of the steering vector of the desired signal makes the output SNR small when the DOA mismatch level is large. For the SMI beamformer, the wrong constraint of $\boldsymbol{w}^{H} \overline{\boldsymbol{a}}=1$ brings out a nulling in the real DOA of the desired signal. Thus, the output SNR is quite small for large DOA mismatch level. The ESB, Capoon-est. beamformer, and Capoon-est-L. beamformer are not very sensitive to the DOA mismatch level. However, due to the constraint between the weight and the estimate steering vector of the desired signal, their output SNR is smaller than the optimal one and the proposed one. Therefore, their performance is inferior to the proposed beamformer.

\section{Conclusions}

A robust beamforming method based on the matrix reconstruction is proposed. In this beamformer, two reconstructed matrices, the interference-plus-noise covariance matrix and the desired signal-plus-noise covariance matrix are used to replace their real sample covariance matrix, respectively. Then, an orthogonal subspace, orthogonal to the interference subspace and including the desired signal subspace, can be obtained based on the principle of the output SINR maximization. Finally, an optimal weight vector can be found by maximizing the output power of the desired signal. This novel beamformer is able to always be a value nearly equal to the optimal value when DOA mismatch has a large uncertainty level and whenever the SNR level of the desired signal is low or high. Moreover, it has an excellent convergence rate. Numerical results demonstrate the effectiveness of the proposed beamfomer compared with some of the existing ones.

\section{Competing interests}

The authors declare that they have no competing interests.

\section{Acknowledgements}

This work is supported by the National Natural Science Foundation of China (No.61301262 and No.61371184), the Applied Basic Research Programs of Sichuan Province (No.2013JY0004) and the Fundamental Research Funds for the Central Universitiesv (No.ZYGX2013J022). 


\section{References}

1. M Wax, Y Anu, Performance analysis of the minimum variance beamformer. IEEE Trans. Signal Process. 44(4), 928-937 (1996)

2. M Wax, Y Anu, Performance analysis of the minimum variance beamformer in the presence of steering vector errors. IEEE Trans. Signal Process. 44(4), 938-947 (1996)

3. HL Van Trees, Optimum Array Processing (Wiley, New York, 2002)

4. $A B$ Gershman, Robust adaptive beamforming in sensor arrays. Int. J. Electron. Commun. 53, 305-314 (1999)

5. Y Han, D Zhang, A recursive Bayesian beamforming for steering vector uncertainties. EURASIP J Adv Signal Process. 2013, 108 (2013)

6. DD Feldman, $L J$ Griffiths, A projection approach for robust adaptive beamforming. IEEE Trans. Signal Process. 42(4), 867-876 (1994)

7. BD Carlson, Covariance-matrix estimation errors and diagonal loading in adaptive arrays. IEEE Trans. Aerosp. Electron. Syst. 24(4), 397-401 (1988)

8. J Li, P Stoica, Z Wang, On robust Capon beamforming and diagonal loading. IEEE Trans. Signal Process. 51(7), 1702-1715 (2003)

9. SA Vorobyov, AB Gershman, ZQ Luo, Robust adaptive beamforming using worst-case performance optimization: a solution to the signal mismatch problem. IEEE Trans. Signal Process. 51(2), 313-324 (2003)

10. RG Lorenz, SP Boyd, Robust minimum variance beamforming. IEEE Trans. Signal Process. 53(5), 1684-1696 (2005)

11. JF Sturm, Using SeDuMi 1.02, a MATLAB toolbox for optimization over symmetric cones. Optimization Methods Software 11-2(1-4), 625-653 (1999)

12. A Hassanien, SA Vorobyov, KM Wong, Robust adaptive beamforming using sequential programming: an iterative solution to the mismatch problem. IEEE Signal Process. Lett. 15, 733-736 (2008)

13. A Khabbazibasmenj, SA Vorobyov, A Hassanien, Robust adaptive beamforming via estimating steering vector based on semidefinite relaxation, in Proceedings of the 44th Asilomar Conference on Signals, Systems and Computers, ed. by SAM (Pacific Grove, CA, 2010), pp. 233-235

14. R Mallipeddi, JP Lie, PN Suganthan, SG Razul, CMS See, Robust adaptive beamforming based on covariance matrix reconstruction for look direction mismatch. Progr. Electromagn. Res. Lett 25, 37-46 (2011)

15. A Khabbazibasmenj, SA Vorobyov, A Hassanien, Robust adaptive beamforming based on steering vector estimation with as little as possible prior information. IEEE Trans Signal Process 60(6), 2974-2987 (2012)

16. Y Gu, A Leshem, Robust adaptive beamforming based on interference covariance matrix reconstruction and steering vector estimation. IEEE Trans. Signal Process. 60(7), 3881-3885 (2012)

17. W Zhang, J Wang, S Wu, Robust Capon beamforming against large DOA mismatch. Signal Process. 93(4), 804-810 (2013)

18. J Zhuang, A Manikas, Interference cancellation beamforming robust to pointing errors. IET Signal Process. 7(2), 120-127 (2011)

19. M Grant, S Boyd, YY Ye, CVX: MATLAB software for disciplined convex programming, June 2014. http://cvxr.com/cvx/

20. RA Monzingo, TW Miller, Introduction to Adaptive Arrays (Wiley, New York, 1980)

doi:10.1186/1687-6180-2014-91

Cite this article as: Xie et al:: A robust adaptive beamforming method based on the matrix reconstruction against a large DOA mismatch. EURASIP Journal on Advances in Signal Processing 2014 2014:91.

\section{Submit your manuscript to a SpringerOpen ${ }^{\circ}$ journal and benefit from:}

- Convenient online submission

Rigorous peer review

- Immediate publication on acceptance

- Open access: articles freely available online

- High visibility within the field

- Retaining the copyright to your article

Submit your next manuscript at $>$ springeropen.com 